\title{
Some identities on Bernoulli and Euler polynomials arising from the orthogonality of Laguerre polynomials
}

Taekyun Kim ${ }^{*}$, Seog-Hoon Rim ${ }^{2}$ DV Dolgy ${ }^{3}$ and Sang-Hun Lee ${ }^{4}$

Correspondence: tkkim@kw.ac.kr 'Department of Mathematics, Kwangwoon University, Seoul,

139-701, Republic of Korea

Full list of author information is

available at the end of the article

\section{Abstract}

In this paper, we derive some interesting identities on Bernoulli and Euler polynomials by using the orthogonal property of Laguerre polynomials.

\section{Introduction}

The generalized Laguerre polynomials are defined by

$$
\frac{\exp \left(-\frac{x t}{1-t}\right)}{(1-t)^{\alpha+1}}=\sum_{n=0}^{\infty} L_{n}^{\alpha}(x) t^{n} \quad(\alpha \in \mathbb{Q} \text { with } \alpha>-1) .
$$

From (1.1), we note that

$$
L_{n}^{\alpha}(x)=\sum_{r=0}^{n} \frac{(-1)^{r}\left(\begin{array}{c}
n+\alpha \\
n-r
\end{array}\right) x^{r}}{r !} \quad(\text { see }[1-3])
$$

By (1.2), we see that $L_{n}^{\alpha}(x)$ is a polynomial with degree $n$. It is well known that Rodrigues' formula for $L_{n}^{\alpha}(x)$ is given by

$$
L_{n}^{\alpha}(x)=\frac{x^{-\alpha} e^{x}}{n !}\left(\frac{d^{n}}{d x^{n}}\left(e^{-x} x^{n+\alpha}\right)\right) \quad(\text { see }[1-3])
$$

From (1.3) and a part of integration, we note that

$$
\int_{0}^{\infty} x^{\alpha} e^{-x} L_{m}^{\alpha}(x) L_{n}^{\alpha}(x) d x=\frac{\Gamma(\alpha+n+1)}{n !} \delta_{m, n},
$$

where $\delta_{m, n}$ is a Kronecker symbol. As is well known, Bernoulli polynomials are defined by the generating function to be

$$
\frac{t}{e^{t}-1} e^{x t}=e^{B(x) t}=\sum_{n=0}^{\infty} B_{n}(x) \frac{t^{n}}{n !} \quad(\text { see }[1-29]),
$$

with the usual convention about replacing $B^{n}(x)$ by $B_{n}(x)$.

2 $2012 \mathrm{Kim}$ et al : licensee Springer. This is an Open Access article distributed under the terms of the Creative Commons Attribution License (http://creativecommons.org/licenses/by/2.0), which permits unrestricted use, distribution, and reproduction in any medium, provided the original work is properly cited. 
In the special case, $x=0, B_{n}(0)=B_{n}$ are called the $n$th Bernoulli numbers. By (1.5), we get

$$
B_{n}(x)=\sum_{l=0}^{n}\left(\begin{array}{l}
n \\
l
\end{array}\right) B_{n-l} x^{l} \quad(\text { see }[1-29]) .
$$

The Euler numbers are defined by

$$
E_{0}=1, \quad(E+1)^{n}+E_{n}=2 \delta_{0, n} \quad(\text { see }[27,28]),
$$

with the usual convention about replacing $E^{n}$ by $E_{n}$.

In the viewpoint of (1.6), the Euler polynomials are also defined by

$$
E_{n}(x)=(E+x)^{n}=\sum_{l=0}^{n}\left(\begin{array}{l}
n \\
l
\end{array}\right) E_{n-l} x^{l} \quad(\text { see }[11-24]) .
$$

From (1.7) and (1.8), we note that the generating function of the Euler polynomial is given by

$$
\frac{2}{e^{t}+1} e^{x t}=e^{E(x) t}=\sum_{n=0}^{\infty} E_{n}(x) \frac{t^{n}}{n !} \quad(\text { see }[15-29]) .
$$

By (1.5) and (1.9), we get

$$
\frac{2}{e^{t}+1} e^{x t}=\frac{1}{t}\left(2-2 \frac{2}{e^{t}+1}\right)\left(\frac{t e^{x t}}{e^{t}-1}\right)=-2 \sum_{n=0}^{\infty}\left(\sum_{l=0}^{n} \frac{E_{l+1}}{l+1}\left(\begin{array}{l}
n \\
l
\end{array}\right) B_{n-l}(x)\right) \frac{t^{n}}{n !} .
$$

Thus, by (1.10), we see that

$$
E_{n}(x)=-2 \sum_{l=0}^{n}\left(\begin{array}{l}
n \\
l
\end{array}\right) \frac{E_{l+1}}{l+1} B_{n-l}(x) .
$$

By (1.7) and (1.8), we easily get

$$
\frac{t}{e^{t}-1} e^{x t}=\frac{t}{2}\left(\frac{2 e^{x t}}{e^{t}+1}\right)+\left(\frac{t}{e^{t}-1}\right)\left(\frac{2 e^{x} t}{e^{t}+1}\right) .
$$

Thus, by (1.12), we see that

$$
B_{n}(x)=\sum_{k=0, k \neq 1}^{n}\left(\begin{array}{l}
n \\
k
\end{array}\right) B_{k} E_{n-k}(x)
$$

Throughout this paper, we assume that $\alpha \in \mathbb{Q}$ with $\alpha>-1$. Let $\mathbb{P}_{n}=\{p(x) \in \mathbb{Q}[x] \mid \operatorname{deg} p(x) \leq$ $n$ \} be the inner product space with the inner product

$$
\langle p(x), q(x)\rangle=\int_{0}^{\infty} x^{\alpha} e^{-x} p(x) q(x) d x
$$


where $p(x), q(x) \in \mathbb{P}_{n}$. From (1.4), we note that $\left\{L_{0}^{\alpha}(x), L_{1}^{\alpha}(x), \ldots, L_{n}^{\alpha}(x)\right\}$ is an orthogonal basis for $\mathbb{P}_{n}$.

In this paper, we give some interesting identities on Bernoulli and Euler polynomials which can be derived by an orthogonal basis $\left\{L_{0}^{\alpha}(x), L_{1}^{\alpha}(x), \ldots, L_{n}^{\alpha}(x)\right\}$ for $\mathbb{P}_{n}$.

\section{Some identities on Bernoulli and Euler polynomials}

Let $p(x) \in \mathbb{P}_{n}$. Then $p(x)$ can be generated by $\left\{L_{0}^{\alpha}(x), L_{1}^{\alpha}(x), \ldots, L_{n}^{\alpha}(x)\right\}$ in $\mathbb{P}_{n}$ to be

$$
p(x)=\sum_{k=0}^{n} C_{k} L_{k}^{\alpha}(x)
$$

where

$$
\begin{aligned}
\left\langle p(x), L_{k}^{\alpha}(x)\right\rangle & =C_{k}\left\langle L_{k}^{\alpha}(x), L_{k}^{\alpha}(x)\right\rangle \\
& =C_{k} \int_{0}^{\infty} x^{\alpha} e^{-x} L_{k}^{\alpha}(x) L_{k}^{\alpha}(x) d x \\
& =C_{k} \frac{\Gamma(\alpha+k+1)}{k !} .
\end{aligned}
$$

From (2.2), we note that

$$
\begin{aligned}
C_{k} & =\frac{k !}{\Gamma(\alpha+k+1)}\left\langle p(x), L_{k}^{\alpha}(x)\right\rangle \\
& =\frac{k !}{\Gamma(\alpha+k+1)} \frac{1}{k !} \int_{0}^{\infty}\left(\frac{d^{k}}{d x^{k}} x^{k+\alpha} e^{-x}\right) p(x) d x \\
& =\frac{1}{\Gamma(\alpha+k+1)} \int_{0}^{\infty}\left(\frac{d^{k}}{d x^{k}} x^{k+\alpha} e^{-x}\right) p(x) d x .
\end{aligned}
$$

Let us take $p(x)=\sum_{m=0, m \neq 1}^{n}\left(\begin{array}{c}n \\ m\end{array}\right) B_{m} E_{n}-m(x) \in \mathbb{P}_{n}$. Then, from (2.3), we have

$$
\begin{aligned}
C_{k} & =\frac{1}{\Gamma(\alpha+k+1)} \int_{0}^{\infty}\left(\frac{d^{k}}{d x^{k}} x^{k+\alpha} e^{-x}\right) \sum_{m=0, m \neq 1}^{n}\left(\begin{array}{c}
n \\
m
\end{array}\right) B_{n} E_{n-m}(x) d x \\
& =\frac{(-1)^{k}}{\Gamma(\alpha+k+1)} \sum_{m=0, m \neq 1}^{n-k} \sum_{l=k}^{n-m}\left(\begin{array}{c}
n \\
m
\end{array}\right)\left(\begin{array}{c}
n-m \\
l
\end{array}\right) B_{m} E_{n-m-l} \frac{l !}{(l-k) !} \int_{0}^{\infty} x^{l+\alpha} e^{-x} d x \\
& =\frac{(-1)^{k}}{\Gamma(\alpha+k+1)} \sum_{m=0, m \neq 1}^{n-k} \sum_{l=k}^{n-m}\left(\begin{array}{c}
n \\
m
\end{array}\right)\left(\begin{array}{c}
n-m \\
l
\end{array}\right) B_{m} E_{n-m-l} \frac{l !}{(l-k) !} \Gamma(l+\alpha+1) \\
& =(-1)^{k} \sum_{m=0, m \neq 1}^{n-k} \sum_{l=k}^{n-m}\left(\begin{array}{c}
n \\
m
\end{array}\right)\left(\begin{array}{c}
n-m \\
l
\end{array}\right) B_{m} E_{n-m-l} \frac{l !}{(l-k) !} \frac{(l+\alpha)(l+\alpha-1) \cdots \alpha}{(\alpha+k)(\alpha+k-1) \cdots \alpha} \\
& =(-1)^{k} n ! \sum_{m=0, m \neq 1}^{n-k} \sum_{l=k}^{n-m} \frac{B_{m}}{m !} \frac{E_{n-m-l}}{(n-m-l) !}\left(\begin{array}{c}
l+\alpha \\
l-k
\end{array}\right) .
\end{aligned}
$$

Therefore, by (2.1) and (2.4), we obtain the following theorem. 
Theorem 2.1 For $n \in \mathbb{Z}_{+}$, we have

$$
\begin{aligned}
& \sum_{m=0, m \neq 1}^{n}\left(\begin{array}{c}
n \\
m
\end{array}\right) B_{m} E_{n-m}(x) \\
& =n ! \sum_{k=0}^{n-k}(-1)^{k}\left(\sum_{m=0, m \neq 1}^{n} \sum_{l=k}^{n-m} \frac{B_{m}}{m !} \frac{E_{n}-m-l}{(n-m-l) !}\left(\begin{array}{l}
l+\alpha \\
l-k
\end{array}\right)\right) L_{k}^{\alpha}(x) .
\end{aligned}
$$

From (1.13), we can derive the following corollary.

Corollary 2.2 For $n \in \mathbb{Z}_{+}$, we have

$$
B_{n}(x)=n ! \sum_{k=0}^{n}(-1)^{k}\left(\sum_{m=0, m \neq 1}^{n-k} \sum_{l=k}^{n-m} \frac{B_{m}}{m !} \frac{E_{n}-m-l}{(n-m-l) !}\left(\begin{array}{c}
l+\alpha \\
l-k
\end{array}\right)\right) L_{k}^{\alpha}(x) .
$$

Let us take $p(x)=\sum_{l=0}^{n}\left(\begin{array}{c}n \\ l\end{array}\right) \frac{E_{l+1}}{l+1} B_{n-l}(x)$. By the same method, we get

$$
\begin{aligned}
C_{k} & =\frac{1}{\Gamma(\alpha+k+1)} \int_{0}^{\infty}\left(\frac{d^{k}}{d x^{k}} x^{k+\alpha} e^{-x}\right) \sum_{l=0}^{n}\left(\begin{array}{l}
n \\
l
\end{array}\right) \frac{E_{l+1}}{l+1} B_{n-l}(x) d x \\
& =\frac{1}{\Gamma(\alpha+k+1)} \sum_{l=0}^{n-k} \sum_{m=0}^{n-l}\left(\begin{array}{l}
n \\
l
\end{array}\right)\left(\begin{array}{c}
n-l \\
m
\end{array}\right) \frac{E_{l+1}}{l+1} B_{n-l-m} \int_{0}^{\infty}\left(\frac{d^{k}}{d x^{k}} x^{k+\alpha} e^{-x}\right) x^{m} d x \\
& =\frac{(-1)^{k}}{\Gamma(\alpha+k+1)} \sum_{l=0}^{n-k} \sum_{m=k}^{n-l}\left(\begin{array}{l}
n \\
l
\end{array}\right)\left(\begin{array}{c}
n-l \\
m
\end{array}\right) \frac{E_{l+1}}{l+1} B_{n-l-m} \frac{m !}{(m-k) !} \Gamma(m+\alpha+1) \\
& =(-1)^{k} \sum_{l=0}^{n-k} \sum_{m=k}^{n-l}\left(\begin{array}{c}
n \\
l
\end{array}\right)\left(\begin{array}{c}
n-l \\
m
\end{array}\right) \frac{m !}{(m-k) !} \frac{E_{l+1}}{(l+1)} B_{n-l-m} \frac{(\alpha+m)(\alpha+m-1) \cdots \alpha}{(\alpha+k)(\alpha+k-1) \cdots \alpha} \\
& =(-1)^{k} n ! \sum_{l=0}^{n-k} \sum_{m=k}^{n-l}\left(\begin{array}{c}
\alpha+m \\
m-k
\end{array}\right) \frac{E_{l+1}}{(l+1) !} \frac{B_{n-l-m}}{(n-l-m) !} .
\end{aligned}
$$

Therefore, by (1.11), (2.1), and (2.5), we obtain the following theorem.

Theorem 2.3 For $n \in \mathbb{Z}_{+}$, we have

$$
-\frac{E_{n}(x)}{2}=n ! \sum_{k=0}^{n}(-1)^{k}\left(\sum_{l=0}^{n-k} \sum_{m=k}^{n-l}\left(\begin{array}{c}
\alpha+m \\
m-k
\end{array}\right) \frac{E_{m+1}}{(m+1) !} \frac{B_{n-m-l}}{(n-m-l) !}\right) L_{k}^{\alpha}(x) .
$$

For $n \in \mathbb{N}$ with $n \geq 2$ and $m \in \mathbb{Z}_{+}$with $n-m \geq 0$, we have

$$
\begin{aligned}
B_{n-m}(x) B_{m}(x)= & \sum_{r}\left\{\left(\begin{array}{c}
n-m \\
2 r
\end{array}\right) m+\left(\begin{array}{l}
m \\
2 r
\end{array}\right)(n-m)\right\} \frac{B_{2 r} B_{n-2 r}(x)}{n-2 r} \\
& +(-1)^{m+1} \frac{(n-m) ! m !}{n !} B_{n} \in \mathbb{P}_{n} \quad(\text { see [8]). }
\end{aligned}
$$


Let us take $p(x)=B_{n-m}(x) B_{m}(x) \in \mathbb{P}_{n}$. Then $p(x)$ can be generated by an orthogonal basis $\left\{L_{0}^{\alpha}(x), L_{1}^{\alpha}(x), \ldots, L_{n}^{\alpha}(x)\right\}$ in $\mathbb{P}_{n}$ to be

$$
p(x)=\sum_{k=0}^{n} C_{k} L_{k}^{\alpha}(x)
$$

From (2.3), (2.6), and (2.7), we note that

$$
\begin{aligned}
& C_{k}= \frac{1}{\Gamma(\alpha+k+1)} \int_{0}^{\infty}\left(\frac{d^{k}}{d x^{k}} x^{k+\alpha} e^{-x}\right) p(x) d x \\
&= \frac{1}{\Gamma(\alpha+k+1)} \sum_{r=0}^{\left[\frac{n}{2}\right]}\left\{\left(\begin{array}{c}
n-m \\
2 r
\end{array}\right) m+\left(\begin{array}{c}
m \\
2 r
\end{array}\right)(n-m)\right\} \\
& \times \frac{B_{2 r}}{n-2 r} \int_{0}^{\infty}\left(\frac{d^{k}}{d x^{k}} x^{k+\alpha} e^{-x}\right) B_{n-2 r}(x) d x \\
&= \frac{1}{\Gamma(\alpha+k+1)} \sum_{r=0}^{\left[\frac{n}{2}\right]}\left\{\left(\begin{array}{c}
n-m \\
2 r
\end{array}\right) m+\left(\begin{array}{c}
m \\
2 r
\end{array}\right)(n-m)\right\} \frac{B_{2 r}}{n-2 r} \\
& \times \sum_{l=0}^{n-2 r}\left(\begin{array}{c}
n-2 r \\
l
\end{array}\right) B_{n-2 r-l} \int_{0}^{\infty}\left(\frac{d^{k}}{d x^{k}} x^{k+\alpha} e^{-x}\right) x^{l} d x \\
& \frac{1}{\Gamma(\alpha+k+1)} \sum_{r=0}^{\left[\frac{n}{2}\right]} \sum_{l=0}^{n-2 r}\left\{\left(\begin{array}{c}
n-m \\
2 r
\end{array}\right) m+\left(\begin{array}{c}
m \\
2 r
\end{array}\right)(n-m)\right\}\left(\begin{array}{c}
n-2 r \\
l
\end{array}\right) \\
& \times \frac{B_{2 r} B_{n-2 r-l}}{n-2 r} \int_{0}^{\infty}\left(\frac{d^{k}}{d x^{k}} x^{k+\alpha} e^{-x}\right) x^{l} d x \\
&= \frac{(-1)^{k}}{\Gamma(\alpha+k+1)} \sum_{r=0}^{\left[\frac{n-k}{2}\right]} \sum_{l=k}^{n-2 r}\left\{\left(\begin{array}{c}
n-m \\
2 r
\end{array}\right) m+\left(\begin{array}{c}
m \\
2 r
\end{array}\right)(n-m)\right\}\left(\begin{array}{c}
n-2 r \\
l
\end{array}\right) \\
& \times \frac{B_{2 r} B_{n-2 r-l} l !}{(n-2 r)(l-k) !} \Gamma(\alpha+l+1) . \\
&\left(\begin{array}{ll}
n-k \\
l
\end{array}\right)
\end{aligned}
$$

It is easy to show that

$$
\begin{aligned}
\frac{\Gamma(\alpha+l+1)}{\Gamma(\alpha+k+1)(l-k) !} & =\frac{(\alpha+l)(\alpha+l-1) \cdots \alpha \Gamma(\alpha)}{(\alpha+k)(\alpha+k-1) \cdots \alpha \Gamma(\alpha)(l-k) !} \\
& =\frac{(\alpha+l)(\alpha+l-1) \cdots(\alpha+k+1)}{(\alpha-k) !}=\left(\begin{array}{c}
\alpha+l \\
l-k
\end{array}\right) .
\end{aligned}
$$

By (2.8) and (2.9), we get

$$
\begin{aligned}
C_{k}= & (-1)^{k} \sum_{r=0}^{\left[\frac{n-k}{2}\right]} \sum_{l=k}^{n-2 r}\left\{\left(\begin{array}{c}
n-m \\
2 r
\end{array}\right) m+\left(\begin{array}{l}
m \\
2 r
\end{array}\right)(n-m)\right\} \\
& \times\left(\begin{array}{c}
n-2 r \\
l
\end{array}\right)\left(\begin{array}{c}
\alpha+l \\
l-k
\end{array}\right) \frac{l ! B_{2 r} B_{n-2 r-l}}{(n-2 r)} .
\end{aligned}
$$

Therefore, by (2.7) and (2.10), we obtain the following theorem. 
Theorem 2.4 For $n \in \mathbb{N}$ with $n \geq 2$ and $m \in \mathbb{Z}_{+}$with $n-m \geq 0$, we have

$$
\begin{aligned}
B_{n-m}(x) B_{m}(x)= & \sum_{k=0}^{n}(-1)^{k}\left\{\sum_{r=0}^{\left[\frac{n-k}{2}\right]} \sum_{l=k}^{n-2 r}\left(\left(\begin{array}{c}
n-m \\
2 r
\end{array}\right) m+\left(\begin{array}{c}
m \\
2 r
\end{array}\right)(n-m)\right)\right. \\
& \left.\times\left(\begin{array}{c}
n-2 r \\
l
\end{array}\right)\left(\begin{array}{c}
\alpha+l \\
l-k
\end{array}\right) \frac{l ! B_{2 r} B_{n-2 r-l}}{(n-2 r)}\right\} L_{k}^{\alpha}(x) .
\end{aligned}
$$

It is easy to show that

$$
\frac{t^{2} e^{t(x+y)}}{\left(e^{t}-1\right)^{2}}=(x+y-1) \frac{t^{2} e^{t(x+y-1)}}{e^{t}-1}-\frac{t^{2} d}{d t}\left(\frac{e^{t(x+y-1)}}{e^{t}-1}\right)
$$

From (2.11), we have

$$
\sum_{k=0}^{n}\left(\begin{array}{l}
n \\
k
\end{array}\right) B_{k}(x) B_{n-k}(y)=(1-n) B_{n}(x+y)+(x+y-1) n B_{n-1}(x+y) \quad(\text { see }[11])
$$

Let $x=y$. Then by (2.12), we get

$$
\sum_{k=0}^{n}\left(\begin{array}{l}
n \\
k
\end{array}\right) B_{k}(x) B_{n-k}(x)=(1-n) B_{n}(2 x)+(2 x-1) B_{n-1}(2 x)
$$

Let us take $p(x)=\sum_{k=0}^{n}\left(\begin{array}{l}n \\ k\end{array}\right) B_{k}(x) B_{n-k}(x) \in \mathbb{P}_{n}$. Then $p(x)$ can be generated by an orthogonal basis $\left\{L_{0}^{\alpha}(x), L_{1}^{\alpha}(x), \ldots, L_{n}^{\alpha}(x)\right\}$ in $\mathbb{P}_{n}$ to be

$$
p(x)=\sum_{k=0}^{n}\left(\begin{array}{l}
n \\
k
\end{array}\right) B_{k}(x) B_{n-k}(x)=\sum_{k=0}^{n} C_{k} L_{k}^{\alpha}(x) .
$$

From (2.3), (2.13), and (2.14), we can determine the coefficients $C_{k}$ 's to be

$$
\begin{aligned}
C_{k}= & \frac{1}{\Gamma(\alpha+k+1)} \int_{0}^{\infty}\left(\frac{d^{k}}{d x^{k}} x^{k+\alpha} e^{-x}\right) p(x) d x \\
= & \frac{1}{\Gamma(\alpha+k+1)}\left\{(1-n) \int_{0}^{\infty}\left(\frac{d^{k}}{d x^{k}} x^{k+\alpha} e^{-x}\right) B_{n}(2 x) d x\right. \\
& \left.+n \int_{0}^{\infty}\left(\frac{d^{k}}{d x^{k}} x^{k+\alpha} e^{-x}\right)(2 x-1) B_{n-1}(2 x) d x\right\} .
\end{aligned}
$$

By simple calculation, we get

$$
\begin{gathered}
\frac{1}{\Gamma(\alpha+k+1)} \int_{0}^{\infty}\left(\frac{d^{k}}{d x^{k}} x^{k+\alpha} e^{-x}\right)(2 x-1) B_{n-1}(2 x) d x \\
=2(-1)^{k} \sum_{l=k-1}^{n-1}\left(\begin{array}{c}
n-1 \\
l
\end{array}\right) 2^{l} B_{n-1-l}\left(\begin{array}{c}
\alpha+l+1 \\
l-k+1
\end{array}\right)(l+1) ! \\
+(-1)^{k+1} \sum_{l=k}^{n-1}\left(\begin{array}{c}
n-1 \\
l
\end{array}\right) 2^{l} B_{n-1-l}\left(\begin{array}{c}
\alpha+l \\
l-k
\end{array}\right) l !
\end{gathered}
$$


and

$$
\begin{gathered}
\frac{1}{\Gamma(\alpha+k+1)} \int_{0}^{\infty}\left(\frac{d^{k}}{d x^{k}} x^{k+\alpha} e^{-x}\right) B_{n}(2 x) d x \\
=(-1)^{k} \sum_{l=k}^{n}\left(\begin{array}{l}
n \\
l
\end{array}\right) 2^{l} B_{n-l} l !\left(\begin{array}{l}
l+\alpha \\
l-k
\end{array}\right) .
\end{gathered}
$$

Therefore, by (2.13), (2.14), (2.15), (2.16), and (2.17), we obtain the following theorem.

Theorem 2.5 For $n \in \mathbb{Z}_{+}$, we get

$$
\begin{aligned}
\sum_{k=0}^{n}\left(\begin{array}{l}
n \\
k
\end{array}\right) B_{k}(x) B_{n-k}(x) \\
=(1-n) \sum_{k=0}^{n}\left\{(-1)^{k} \sum_{l=k}^{n}\left(\begin{array}{l}
n \\
l
\end{array}\right) 2^{l} B_{n-l} l !\left(\begin{array}{c}
l+\alpha \\
l-k
\end{array}\right)\right\} L_{k}^{\alpha}(x) \\
+n \sum_{k=0}^{n}(-1)^{k}\left\{\sum_{l=k-1}^{n-1}\left(\begin{array}{c}
n-1 \\
l
\end{array}\right) 2^{l+1} B_{n-1-l}(l+1) !\left(\begin{array}{c}
\alpha+l+1 \\
l-k+1
\end{array}\right)\right. \\
\left.\quad-\sum_{l=k}^{n-1}\left(\begin{array}{c}
n-1 \\
l
\end{array}\right) 2^{l} B_{n-l-1} l !\left(\begin{array}{c}
\alpha+l \\
l-k
\end{array}\right)\right\} L_{k}^{\alpha}(x) .
\end{aligned}
$$

\section{Competing interests}

The authors declare that they have no competing interests.

\section{Authors' contributions}

All authors contributed equally to the manuscript and typed, read, and approved the final manuscript.

\section{Author details}

${ }^{1}$ Department of Mathematics, Kwangwoon University, Seoul, 139-701, Republic of Korea. ${ }^{2}$ Department of Mathematics Education, Kyungpook National University, Taegu, 702-701, Republic of Korea. ${ }^{3}$ Hanrimwon, Kwangwoon University, Seoul, 139-701, Republic of Korea. ${ }^{4}$ Division of General Education, Kwangwoon University, Seoul, 139-701, Republic of Korea.

\section{Acknowledgements}

The authors would like to express their deep gratitude to the referees for their valuable suggestions and comments.

Received: 8 August 2012 Accepted: 6 November 2012 Published: 22 November 2012

\section{References}

1. Carlitz, L: An integral for the product of two Laguerre polynomials. Boll. Unione Mat. Ital. 17(17), 25-28 (1962)

2. Carlitz, L: On the product of two Laguerre polynomials. J. Lond. Math. Soc. 36, 399-402 (1961)

3. Cangul, N, Kurt, V, Ozden, H, Simsek, Y: On the higher-order w-q-Genocchi numbers. Adv. Stud. Contemp. Math. 19(1), 39-57 (2009)

4. Akemann, G, Kieburg, M, Phillips, MJ: Skew-orthogonal Laguerre polynomials for chiral real asymmetric random matrices. J. Phys. A 43(37), Art. ID 375207 (2010)

5. Bayad, A, Kim, T: Identities for the Bernoulli, the Euler and the Genocchi numbers and polynomials. Adv. Stud. Contemp. Math. 20(2), 247-253 (2010)

6. Bayad, A: Modular properties of elliptic Bernoulli and Euler functions. Adv. Stud. Contemp. Math. 20(3), 389-401 (2010)

7. Carlitz, L: Note on the integral of the product of several Bernoulli polynomials. J. Lond. Math. Soc. 34, 361-363 (1959)

8. Carlitz, L: Some generating functions for Laguerre polynomials. Duke Math. J. 35, 825-827 (1968)

9. Costin, RD: Orthogonality of Jacobi and Laguerre polynomials for general parameters via the Hadamard finite part. J. Approx. Theory 162(1), 141-152 (2010)

10. Ding, D, Yang, J: Some identities related to the Apostol-Euler and Apostol-Bernoulli polynomials. Adv. Stud. Contemp. Math. 20(1), 7-21 (2010)

11. Hansen, ER: A Table of Series and Products. Prentice Hall, Englewood Cliffs (1975) 
12. Kudo, A: A congruence of generalized Bernoulli number for the character of the first kind. Adv. Stud. Contemp. Math. 2, 1-8 (2000)

13. Kim, T, Choi, J, Kim, YH, Ryoo, CS: On q-Bernstein and q-Hermite polynomials. Proc. Jangjeon Math. Soc. 14(2), 215-221 (2011)

14. Kim, T: A note on q-Bernstein polynomials. Russ. J. Math. Phys. 18(1), 73-82 (2011)

15. Kim, $\mathrm{T}$ : Some identities on the $q$-Euler polynomials of higher order and $q$-Stirling numbers by the fermionic $p$-adic integral on $\mathbb{Z}_{p}$. Russ. J. Math. Phys. 16(4), 484-491 (2009)

16. Kim, T: Symmetry of power sum polynomials and multivariate fermionic $p$-adic invariant integral on $\mathbb{Z}_{p}$. Russ. J. Math Phys. 16(1), 93-96 (2009)

17. Ozden, $\mathrm{H}$, Cangul, IN, Simsek, Y: Multivariate interpolation functions of higher-order $q$-Euler numbers and their applications. Abstr. Appl. Anal. 2008, Art. ID 390857 (2008)

18. Choi, J, Kim, DS, Kim, T, Kim, YH: Some arithmetic identities on Bernoulli and Euler numbers arising from the $p$-adic integrals on $\mathbb{Z}_{p}$. Adv. Stud. Contemp. Math. 22(2), 239-247 (2012)

19. Ozden, H, Cangul, IN, Simsek, Y: Remarks on q-Bernoulli numbers associated with Daehee numbers. Adv. Stud. Contemp. Math. 18(1), 41-48 (2009)

20. Rim, SH, Lee, SJ: Some identities on the twisted $(h, q)$-Genocchi numbers and polynomials associated with q-Bernstein polynomials. Int. J. Math. Math. Sci. 2011, Art. ID 482840 (2011)

21. Rim, SH, Bayad, A, Moon, EJ, Jin, JH, Lee, SJ: A new construction on the q-Bernoulli polynomials. Adv. Differ. Equ. 2011, 34 (2011)

22. Rim, SH, Jin, JH, Moon, EJ, Lee, SJ: Some identities on the q-Genocchi polynomials of higher-order and q-Stirling numbers by the fermionic $p$-adic integral on $\mathbb{Z}_{p}$. Int. J. Math. Math. Sci. 2010, Art. ID 860280 (2010)

23. Ryoo, CS: On the generalized Barnes type multiple $q$-Euler polynomials twisted by ramified roots of unity. Proc. Jangjeon Math. Soc. 13(2), 255-263 (2010)

24. Ryoo, CS: A note on the Frobenius-Euler polynomials. Proc. Jangjeon Math. Soc. 14(4), 495-501 (2011)

25. Ryoo, CS: Some relations between twisted $q$-Euler numbers and Bernstein polynomials. Adv. Stud. Contemp. Math. 21(2), 217-223 (2011)

26. Ryoo, CS: Some identities of the twisted $q$-Euler numbers and polynomials associated with $q$-Bernstein polynomials Proc. Jangjeon Math. Soc. 14(2), 239-248 (2011)

27. Simsek, Y: Generating functions of the twisted Bernoulli numbers and polynomials associated with their interpolation functions. Adv. Stud. Contemp. Math. (Kyungshang) 16(2), 251-278 (2008)

28. Simsek, Y: Special functions related to Dedekind-type DC-sums and their applications. Russ. J. Math. Phys. 17(4), 495-508 (2010)

29. Simsek, Y: On $p$-adic twisted $q$-L-functions related to generalized twisted Bernoulli numbers. Russ. J. Math. Phys. 13(3), 340-348 (2006)

doi:10.1186/1687-1847-2012-201

Cite this article as: Kim et al.: Some identities on Bernoulli and Euler polynomials arising from the orthogonality of Laguerre polynomials. Advances in Difference Equations 2012 2012:201.

\section{Submit your manuscript to a SpringerOpen ${ }^{\circ}$ journal and benefit from:}

- Convenient online submission

Rigorous peer review

- Immediate publication on acceptance

- Open access: articles freely available online

- High visibility within the field

- Retaining the copyright to your article 